\title{
Economic development, social integration, and the subjective well-being of residents in the western regions of China: based on the perspective of multi- ethnic China
}

\author{
Jianke Yang ${ }^{1} \cdot$ Qi Wang ${ }^{1}$
}

Received: 12 May 2020 / Accepted: 28 February 2021 / Published online: 25 March 2021

(c) The Author(s). 2021

\begin{abstract}
Based on a large sample of survey data collected from 12 Chinese provinces, municipalities directly under the central government and autonomous regions in the western regions of China, this article discusses the subjective well-being of the residents of all ethnic groups in the western regions, along the two dimensions of economic development and social integration. The results show that the overall subjective well-being of all ethnic groups in Western China is good, and the percentage of people who indicated that they often feel happy is generally higher than the percentage of those who often feel unhappy. The residents of ethnic minority status such as the Huis and the Uyghurs experience good subjective well-being more often than residents of other ethnic groups. Microscopic indicators, such as the level of household income, and macroscopic indicators, such as the level of regional economic development, can exert significant positive impacts on the subjective wellbeing of residents from various ethnic groups. In addition, emotional integration, trust-based integration, and integration through communication-as important variables of social identity and social acceptance — can also have significant positive impacts on improving the subjective well-being of residents of all ethnic groups. The article concludes that, for the residents of all ethnic groups living in the less developed western regions of China, economic development and increased income remain the key means of improving the subjective well-being, and promoting inter-
\end{abstract}

Jianke Yang

jkyang@mail.xjtu.edu.cn

Qi Wang

yuji5712@163.com

1 Department of Sociology, School of Humanities, Xi'an Jiaotong University, 28 Xianning West Road, Beilin District, Xi'an City 710049, Shaanxi Province, China 
ethnic interactions and strengthening social integration are also positive methods of effectively improving the subjective well-being.

Keywords Subjective well-being of ethnic groups $\cdot$ Economic development $\cdot$ Social integration · China

\section{Abbreviations}

GDP: $\quad$ Gross Domestic Product

CSSC: China Survey of Social Change

CGSS: Chinese General Social Survey

CCTV2: the China Central Television financial channel

\section{Background}

The western region of China is the most important multi-ethnic region in China. While it is relatively underdeveloped in economy, the ethnic minorities in the West have their own cultural traditions, religious beliefs and lifestyle. It is an important task for China to build a well-off society in an all-round way. After 40 years of economic development and social transformation since the beginning of China's reform and opening up, what is the subjective well-being status of the residents? Are there differences in their subjective well-being of different ethnic groups? Do economic and cultural variables have the same impact on the subjective well-being of different ethnic groups? Does the "Happiness-income Paradox" proposed by R. Easterlin apply in the western ethnic regions of China? The answers to these questions have direct bearing upon the development and social harmony of the western ethnic regions. To obtain the answers, focusing on the subjective well-being of ethnic groups in the western regions of China, ${ }^{1}$ this article specifically analyzes how factors like economic development and social integration influence the variations in the subjective well-being of residents from various ethnic groups, which aims to provide theoretical and practical reference for enhancing the subjective well-being of ethnic groups and promoting ethnic development and solidarity among ethnic groups.

It is significant to study subjective well-being from an ethnic perspective, because the improvement of community and individual subjective well-being represents an important part in improving quality of life for an entire social community. Although individual psychology can directly explain variations in their subjective well-being, how do people's living conditions affect the generation and change of subjective well-being? How do different ethnic cultures create different experiences of happiness? At present, academic research on the subject mainly focuses on the following two aspects.

The first approach tends to focus on comparative studies looking at subjective well-being among different ethnic groups. Of all the arguments surrounding

\footnotetext{
${ }^{1}$ In addition to the Han ethnic group, the samples used in this article involve 28 ethnic minorities in China. In order to streamline the analysis process, these ethnic minorities are divided into a number of groups based on the principle of proximity.
} 
subjective well-being among ethnic groups, the assertion that "the ethnic majority experiences more happiness than people of ethnic minorities" is among the most widely debated. Studies have found a significant correlation between ethnic groups and subjective well-being. For instance, black people score significantly lower than their white peers when it comes to subjective well-being with regard to family life, occupation and work. Compared with physical or health-related factors, racial factors can effectively explain much of the variation between people's subjective well-being (Saunders, 2013). A survey on the levels of subjective well-being of British Canadians and Chinese Canadians showed that racial differences significantly affect personal living standards and developmental achievements (Spiers, Andrew, Walker, Gordon, \& J, 2009). Psychological research conducted by Chinese scholars on the subjective well-being of Han and ethnic minority college students found that, while ethnic minority college students generally have moderately high subjective well-being, they are significantly higher than their Han contemporaries in terms of negative emotion scores. (Zhang, 2008) In a general sense, people tend to believe that the ethnic majority (generally defined as the ethnic group with the absolute majority of a country's population, or when the national law, administration, and the social and public life are based on the language, writing and culture of an ethnic group) occupies a dominant position in the society and has access to resources related to politics, economy and culture, while ethnic minorities are most often found at the margins of a society. Individuals of ethnic minority backgrounds are gradually inclined to make negative self-assessment, and therefore may make negative self-evaluations. However, the research findings of some Chinese scholars show that the subjective well-being of the Han Chinese is not all higher than that of the people of ethnic minorities. Some ethnic minorities have lower subjective well-being than the Han nationality, while others, such as Uygur and Hui, their proportion of self-reported happiness is far higher than that of the Hans (Xiong, 2014). A survey of teachers in Xinjiang found that Uighur and Kazakh teachers are significantly higher than the Han teachers in terms of both their emotional index and their overall subjective well-being (Guo, 2008).

The second approach places its focus on exploratory studies looking at the factors influencing the subjective well-being within ethnic groups. Some scholars believe that the variations in subjective well-being among different ethnic groups may be caused by differences in their values and goals (Diener, 2003). A study based in South Africa found that residents of different ethnic groups have different conceptual definitions of subjective well-being, and ethnic culture bears a strong correlation to the level of subjective well-being (Daniel, 2007). Some scholars found that economic development exerts different impacts on the subjective wellbeing of different residents in China's ethnic regions. Zhao Changlin's analysis of the factors influencing people's subjective well-being in 17 ethnic regions in Yunnan Province found that, with an increase of income, residents felt an increase in subjective well-being. However, in general, the impact of income on subjective well-being was an initial increase, followed by a decrease. This trend was attributed to the ability of the residents to increase their income and the level of their expectations (Zhao, 2011). An investigation on Lanzhou City (a city in northwest China) conducted by Yang Chaojie and others found that the level of economic 
development does not have a significant impact on the subjective well-being of ethnic groups. The unique religious beliefs and ethnic cultures in these regions bring great spiritual satisfaction to local people. As a result, people have less materialistic desires, and the level of income bears a less significant impact on the level of subjective well-being of ethnic groups in these regions (Yang, 2015). Abraham Harold Maslow's hierarchy of needs theory maintains that, with the improvement of economic conditions, people are no longer satisfied with improved material conditions; instead they try to fulfill needs at higher levels such as social recognition and selfrealization. In recent years, some Chinese scholars have focused on other factors influencing the subjective well-being of ethnic groups other than economic variables. Some studies pointed out that, compared with Han university students, ethnic minority university students have only a moderate degree of subjective well-being. Due to the adverse effects of factors such as cultural differences and lower university admission standards on their psychological well-being, social support plays a particularly important role in managing the subjective well-being of ethnic minority university students (Zhou, 2007). However, this research finding is specific to university students, and it remains to be seen whether the results can be applied to ethnic minorities as a whole.

In sum, although scholars have conducted exploratory research on many aspects of the subjective well-being of ethnic groups, there are still shortcomings to the existing research. First, most of the current research on the subjective well-being of ethnic groups make inadequate theoretical analysis of the causal mechanisms at play. Second, it is hard for many studies to display the overall situation of the subjective well-being of ethnic groups since they solely focus on a single ethnic group or a specific region with limited survey data, rendering them incapable of systematically exploring the theoretical logic of the factors influencing the subjective well-being of residents from various ethnic groups. Third, no comparative analysis is made to clarify the unity and differences regarding the subjective well-being between residents of different ethnic groups. Therefore, taking the subjective well-being of ethnic groups in the western regions of China as its research target and basing its analysis on a large sample of survey data collected from 12 provinces, direct-administered municipalities and autonomous regions, this article emphatically discusses the relationships between the subjective well-being of ethnic groups and factors such as economic development and social integration, and reveals the overall conditions and structural characteristics of the subjective well-being of ethnic groups living in the western regions of China through empirical analysis.

\section{Data and methods}

\section{Data}

The data used in this paper to analyse the subjective well-being of various Western Chinese ethnic groups comes from the 2010 China Survey of Social Change (CSSC) project, which was jointly organized by the Institute for 
Empirical Social Science Research, Xi'an Jiaotong University and other institutions. This project was a large-scale, household questionnaire survey conducted through stratified random sampling, covering 12 western provinces, autonomous regions and direct-administered municipalities. The survey respondents were adults over 18 years old. The final number of valid samples in this article was 10,098 . Nearly $30 \%$ of the total samples were residents of ethnic minorities, and the ethnic minority population in the western region accounted for nearly $80 \%$ of the total national ethnic minority population. Such percentages provide a good database for analyzing the subjective well-being of ethnic groups in China.

\section{Classification and measurement of ethnic groups}

In order to ensure succinctness and focus, this paper classifies 28 ethnic groups randomly sampled in the survey into four categories by performing clustering and group analysis based on the principle of proximity between ethnic minorities and taking into account factors such as language and region. These four categories are as follows: (1) the people of Manchu, Mongolian, $\mathrm{Tu}$, and Daur ethnic groups are clustered and given the shortened title "the Manchu-Mongol ethnic cluster", as their languages are close to each other and belong to the Altaic language family; (2) the people of Hui, Uyghur, Kazakh, Kirgiz, and Dongxiang ethnic minorities are clustered and given the title "the Hui-Uyghur ethnic cluster" because of their geographical proximity in the northwestern region; (3) the Tibetans, and the peoples of Yi, Tujia, Dai, Bai, Hani, Lahu, and Naxi ethnic minorities are clustered and called "the TibetanYi ethnic cluster" because of their closely related languages which belong to the Tibetan-Burmese branch of the Sino-Tibetan language family; (4) the people of Zhuang, Dai, Buyi, Dai, Shui, Li, Gelao, Mulao, Miao, Yao, and Jing ethnic groups are clustered and given the label "the Zhuang and Dong/ Miao and Yao ethnic cluster"as they mostly live in the southwestern region and their languages are closely related, essentially all belonging to the ZhuangDong or Miao-Yao branch of the Sino-Tibetan language family. The people of the above four ethnic groups plus the Hans form the five groups clustered for the purpose of the research (Table 1). This classification is similarly applied in Bian Yanjie's research on the subjective well-being of Chinese residents (Bian, 2014).

To corroborate the above classification, this article also makes a correlation analysis between these five major ethnic groups and their religious beliefs as an auxiliary basis for the classification. ${ }^{2}$ The methods used to classify the five

\footnotetext{
${ }^{2}$ The "religious beliefs" are divided into six types, with the inclusion of the percentage of non-believers in addition to the percentages of believers of main religions. The data on the percentages of different religious beliefs in the total population was obtained through questionnaire surveys and calculations done by the research group. The information about religious belief of each individual is obtained based on the subjective identification of the individual through face-to-face interview.
} 
Table 1 The Classification of Ethnic Groups in Western China

\begin{tabular}{|c|c|c|}
\hline Classification & Religion/Language & Ethnic Groups Studied ${ }^{2}$ \\
\hline $\begin{array}{l}\text { Manchu, Mongolian and other } \\
\text { ethnic groups }\end{array}$ & the Altai language family & Manchu, Mongolian, Tu, and Daur \\
\hline $\begin{array}{l}\text { Hui, Uygur and other ethnic } \\
\text { groups }\end{array}$ & Islam & $\begin{array}{l}\text { Hui, Uygur, Kazakh, Kirgiz, and } \\
\text { Dongxiang }\end{array}$ \\
\hline $\begin{array}{l}\text { the Zhuang, Dong, Miao and } \\
\text { Yao and other ethnic groups }\end{array}$ & $\begin{array}{l}\text { Zhuang-Dong or Miao- } \\
\text { Yao language branch }\end{array}$ & $\begin{array}{l}\text { Zhuang, Dong, Buyi, Dai, Shui, Li, } \\
\text { Gelao, Mulao, Miao, Yao, and Jing }\end{array}$ \\
\hline $\begin{array}{l}\text { the Tibetan, Yi and other ethnic } \\
\text { groups }\end{array}$ & $\begin{array}{l}\text { Tibetan-Burmese } \\
\text { language branch }\end{array}$ & $\begin{array}{l}\text { Tibetan, Yi, Tujia, Qiang, Bai, Hani, } \\
\text { Lahu, and Naxi }\end{array}$ \\
\hline
\end{tabular}

Note: The total effective sample size is $\mathrm{N}=10,098$

${ }^{2}$ We selected samples by using the stratified random sampling method. We entrusted one university in each of 12 provinces, municipalities directly under the central government, and autonomous regions in the western regions to complete a sample survey, and randomly selected 800-1000 samples from these 12 universities. The final data is the sum of these samples. As the samples of these 28 ethnic minorities were randomly selected, no samples were included from any other ethnic minorities.

major ethnic groups are found to be highly consistent with the alignment of their beliefs. The specific research findings are displayed in Table 2.

It can be seen from the above tables that the Manchu-Mongol ethnic cluster mainly believe in Buddhism (37.20\%) or do not believe in religion (58.94\%); the Hui-Uyghur ethnic cluster share a highly concentrated belief in Islam (93.84\%); ethnic residents including Tibetans and $\mathrm{Yi}$ people either believe in Buddhism $(72.73 \%)$ or do not believe $(24.96 \%)$ in any religion; most the Zhuang and Dong/ Miao and Yao ethnic cluster are non-believers (80.04\%), and the Hans are also basically all non-believers $(85.10 \%)$. These findings reveal a certain isomorphism between religious beliefs and ethnic groups.

\section{The current status and differences between the subjective well-being of various ethnic groups in the western regions of China}

In using the data from the 2010 CSSC, this article re-named the original data categories of "often and sometimes feel happy" and "seldom or never feel happy" as

Table 2 Correlation Analysis on Ethnic Groups and Their Religious Beliefs

\begin{tabular}{|c|c|c|c|c|c|c|}
\hline \multirow[t]{2}{*}{ Ethnic Groups } & \multicolumn{6}{|c|}{ Religious Belief (\%) } \\
\hline & Islam & Christianity & Taoism & Buddhism & $\begin{array}{l}\text { Folk } \\
\text { Religion }\end{array}$ & $\begin{array}{l}\text { No } \\
\text { religion }\end{array}$ \\
\hline the Hans & 0.10 & 2.26 & 0.85 & 9.63 & 2.07 & 85.10 \\
\hline $\begin{array}{l}\text { Manchu, Mongolian and other ethnic } \\
\text { groups }\end{array}$ & 1.45 & 2.42 & 0.00 & 37.20 & 0.00 & 58.94 \\
\hline Hui, Uygur and other ethnic groups & 93.84 & 0.26 & 0.00 & 2.23 & 0.39 & 3.28 \\
\hline Tibetan, Yi and other ethnic groups & 0.08 & 0.08 & 1.12 & 72.73 & 1.04 & 24.96 \\
\hline $\begin{array}{l}\text { Zhuang, Dong, Miao and Yao and other } \\
\text { ethnic groups }\end{array}$ & 0.18 & 0.35 & 1.05 & 9.11 & 9.28 & 80.04 \\
\hline
\end{tabular}

Note: Pearson $\operatorname{chi} 2(20)=1.3 * 104 \operatorname{Pr}=0.00$ 
The state of the subjective well-being of various ethnic groups in the western region $(\%)$

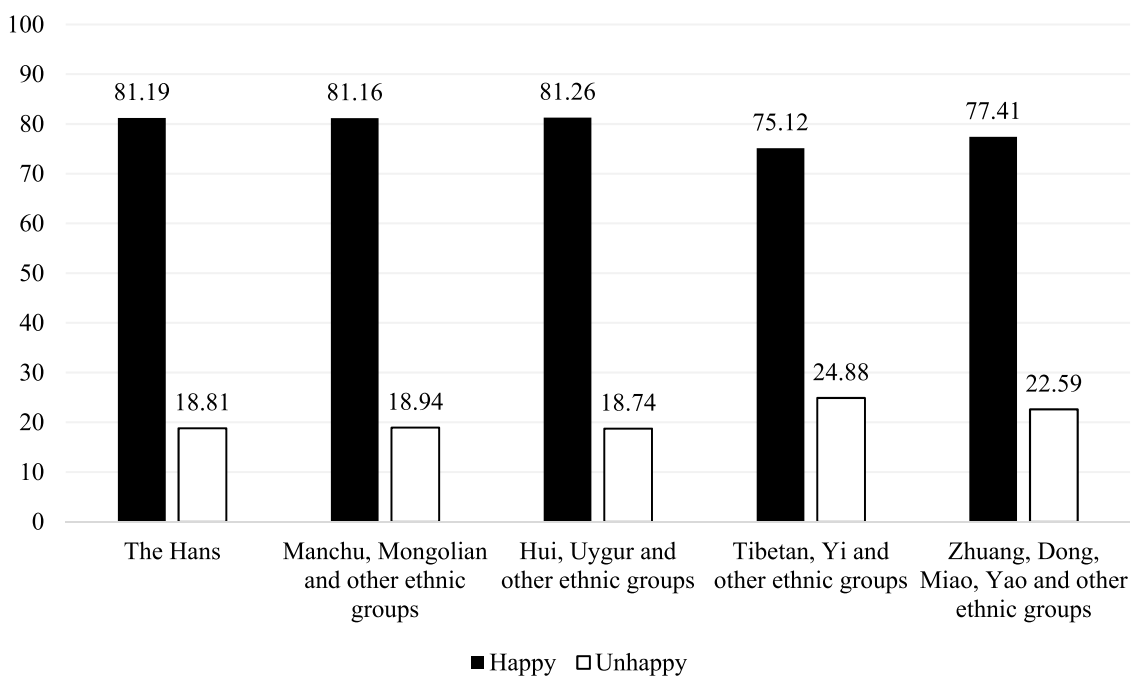

Fig. 1 Bar Graph showing the current state of the subjective well-being of ethnic minority residents in the western regions of China

"happy" and "unhappy" respectively. We then rearranged the data according to ethnic variables, and displayed the proportion of happy people to unhappy people in Fig. 1. As shown in Fig. 1, people of all ethnic groups in the western regions of China feel happy on the whole, and there are far more residents who feel happy than those who feel unhappy (Fig. 1). The percentage of residents who expressed happiness on the survey is over $75 \%$, which is close to the national data from the 2010 Chinese General Social Survey (CGSS) project conducted around the same time $(72.41 \%$ of Han residents and $73.75 \%$ of ethnic minority residents responded that they feel happy). Generally speaking, people of all ethnic groups in the western regions indicated that they feel happy, with a relatively balanced distribution of subjective well-being.

To further understand the subjective well-being of the ethnic residents in the western regions, we have used a line chart to demonstrate the proportion of those who indicated that they often feel happy, compared to those who often feel unhappy (Fig. 2). As seen in Fig. 2, the percentage of those who often feel happy is generally higher than that of those who often feel unhappy, but people of different ethnic groups vary considerably in their subjective well-being. The highest percentage of subjective well-being is seen among the Hui-Uyghur ethnic cluster, followed by the Hans, and the Manchu-Mongol ethnic cluster. In comparison, relatively low percentages of subjective well-being are seen among people of the Zhuang and Dong/Miao and Yao ethnic cluster, and Tibetans and the Yi people. In general, when compared with the Hans, the Hui-Uyghur ethnic cluster indicated higher 


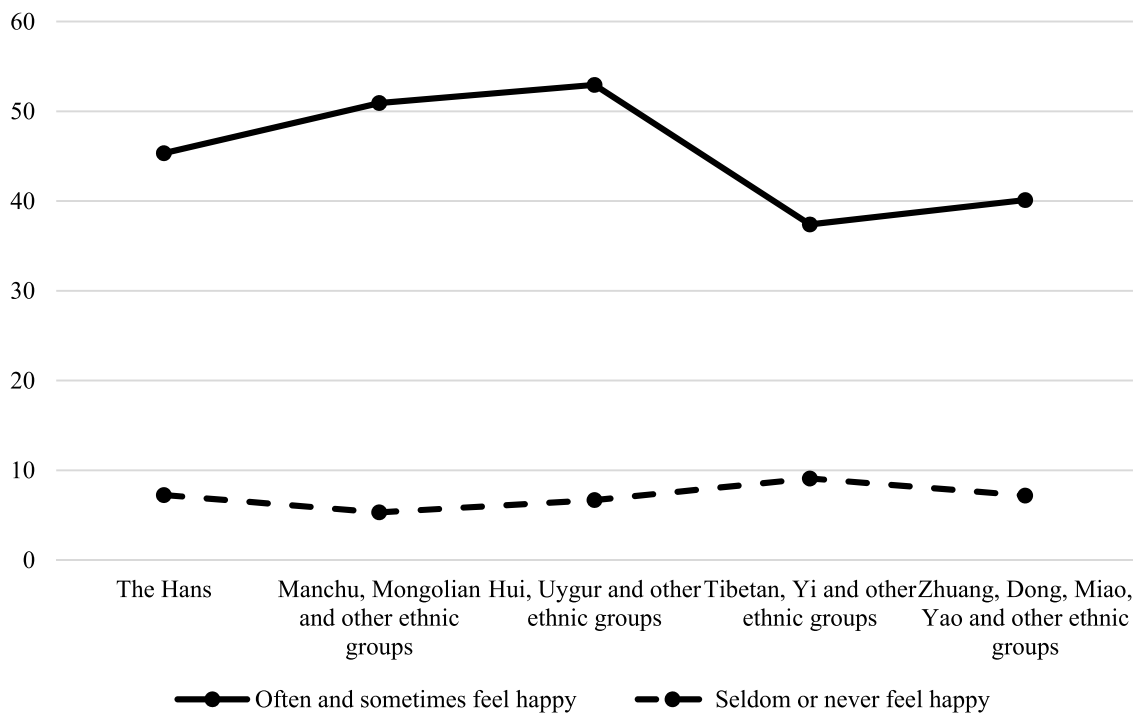

Fig. 2 Line graph showing the ethnic groups who indicated that they often feel happy or unhappy in the western regions of China

levels of subjective well-being while that of the Tibetans-Yi ethnic cluster was lower.

By comparing Fig. 1 and Fig. 2, we can see clearly that the ethnic residents in the western regions of China vary in terms of their subjective well-being. The subjective well-being of the five major ethnic groups can be divided into three levels, with the Hui-Uyghur ethnic cluster at the highest level, the Hans and the ManchuMongol ethnic cluster in the middle level, and ethnic residents including Tibetans, the Yi, Zhuang, Dong, Miao and Yao peoples at the lowest level. Among the people who indicated that they feel happy, the percentage of those who indicated the highest level of happiness (52.95\%) is nearly 16\% higher than that of those who indicated the lowest level (37.40\%). Among the people who indicated that they feel unhappy, the percentage of those at the lowest level $(9.09 \%)$ is more than $2 \%$ higher than the percentage of those who feel happy at the highest level $(6.68 \%)$. However, further analysis is required to ascertain the main reason accounting for these variations in the subjective well-being of different ethnic groups-the unique cultural structure of the ethnic group itself or the role of the external economic and social environment.

\section{Theoretical assumptions and related analysis}

\section{Hypothesis of economic development}

In the past, academic studies looking at subjective well-being tended to focus on discussing the effects of income growth and economic development on 
subjective well-being. The well-known Easterlin Paradox in the study of happiness suggests that economic growth does not necessarily always improve people's subjective well-being. When the economy develops to a certain degree, subjective well-being may stagnate or even decline (Easterlin, 2010). This conclusion has also been confirmed in multinational studies. According to E. Diener, improved income has a short-term, but no obvious or long-term, effect on enhancing people's subjective well-being (Diener, 2002). In his study of the relationship between income and subjective well-being, Chinese scholar Xing Zhanjun found a positive correlation between income and the subjective well-being of urban residents in China. The subjective well-being of highincome groups is significantly higher than that of low-income groups; moreover, the wealth of a given region also influences the correlation between the two groups' subjective well-being (Xing, 2011). Liu Junqiang's analysis, based on 10 years spent tracking the data from the CGSS, also concluded that economic growth and personal income are still the main driving forces for enhancing subjective well-being (Liu, 2012). In addition, new findings were made based on big data investigations. According to the latest survey results released by CCTV2 (the China Central Television financial channel) in 2016:

Money is not everything. The annual household income of RMB300 thousand (about USD 45 thousand) has become the point of inflection. The family members of a household earning more than RMB300 thousand may not necessarily feel happy. It is also found that high-income groups with an annual income of more than RMB1 million (about USD 149 thousand) are less happy than families with an annual income ranging from RMB80 thousand (about USD 12 thousand) to RMB120 thousand (about USD 18 thousand) (CCTV, 2017).

Both sides of the Easterlin Paradox have been verified in China. In the western ethnic regions with special economic, social and cultural conditions, how does the Easterlin Paradox manifest itself? Compared with the central and eastern regions of China, the current economic development of the western ethnic regions lags behind, and economic factors should be the key factors influencing the sense of the happiness of the local people.

Therefore, this article proposes the hypothesis that, from both a macroscopic and microscopic perspective, economic development does influence the subjective well-being of people in the western regions. The hypothesis holds that the higher the degree of economic development and the higher the household income of an ethnic region, the higher the subjective well-being of the local ethnic residents living there.

\section{Hypothesis of social integration}

As a subjective feeling, the formation and evolution of somebody's subjective well-being is a complicated psychological experience. Social influences are equally important as economic conditions. As ethnic residents live in a 
socialized context, their integration with society plays a key role in assessing their psychological sentiments, social cognition, and attitude evaluation. American sociologist Robert Ezra Park (1864-1944) used the term "social distance" to locate social integration, arguing that social distance exists in the understanding and the closeness degree of interpersonal, intergroup relations, and the size of social distance represents the depth of social integration. Later, Bogardus designed the Social Distance Scale as a way of measuring social integration, promoting empirical research on social integration. Social integration takes two forms: the social integration of groups from the macroscopic perspective, and the social integration of individuals from the microscopic perspective. At either level, social identity and social acceptance are the internal psychological mechanisms for social integration, as social identity addresses the issue of positioning and social acceptance addresses that of integration. Social identity and social acceptance between peers are important for the social interactions and psychological health of individuals. Through social identity and social acceptance, individuals can not only share values and experience emotions together, but also select actions more effectively in a way that suits their accepted values and goals (Huang, 2010). It is based on this that positive and happy inner experiences are constructed and inspired. Empirical research by Carlo Klei found that improved social integration significantly impacts people's subjective well-being (Klei, 2013). Studies on migrants conducted by Ji Yongbao and others also point out that migrants experience enhanced subjective well-being as their integration with society deepens, and that the role of social integration is more important than economic integration ( $\mathrm{Ji}, 2016$ ). On the one hand, people of China's ethnic groups have their own specific ethnic culture, lifestyle, and characteristics. On the other hand, people of China's different ethnic groups live in a mixed way; they settle among each other, in some places forming compact communities. This way of living influences the social lives of ethnic residents in terms of their communication, interactions and social integration. In the western regions of China, multi-ethnic groups live together, which have seen relatively under-developed and differentiated local socio-economic development. Under such realistic conditions, if the residents of each ethnic group can achieve better social integration, they will be able to derive more emotional support in their social lives, be able to further expand their networks of social exchange and social support, and be able to enhance mutual trust in their interactions so as to achieve social recognition and social acceptance. In addition, social identity and social acceptance can dispel loneliness and anxiety among residents, and strengthen their sense of security and belongings. Social integration can generate strong positive emotions for residents from all ethnic groups, and therefore plays a positive role in reducing social distance and social exclusion, enhancing solidarity among people of all ethnic groups, and improving the subjective well-being of residents of all ethnic groups.

Marriage is an important variable of the research on subjective well-being. It is widely acknowledged that married residents feel happier than unmarried residents. Marriage can provide familial and emotional comfort to people from 
all ethnic groups. Being married helps to buffer individual life stress, and provides an important source of an individual's sense of belonging and support. Marriage can effectively raise the level of subjective well-being as it provides individual residents with all-round support, including emotional and economic support. The degree of social trust reflects the amount of confidence an individual has in others as well as their perceived social security. Due to the fact that trust can simplify social interactions among residents, effectively reduce the cost of communication and relieve anxiety, it is an important embodiment of social identity of ethnic minority residents. Social networks are pathways that allow residents to expand their social connections and obtain social support, reflecting the social capitals that are owned and at the disposal of the residents. The size of social networks for ethnic minorities and differences in other aspects indicate the degree to which residents obtain information and seek material and emotional support from their social networks.

Therefore, this article examines the social integration of ethnic residents along the dimensions of emotional integration, trust-based integration and integration through communication. Based on such an examination, the article proposes a social integration hypothesis on subjective well-being. The proposal is that the higher the degree of social integration of ethnic residents, the higher their subjective wellbeing. Specifically, married ethnic residents with a higher degree of social trust and a higher degree of heterogeneity in their social networks have higher subjective well-being.

\section{Correlation analysis of the factors influencing the subjective well-being of ethnic residents}

Empirical proof is required to prove whether the theoretical analysis and research hypothesis discussed above are tenable, which use two aspects and five dimensions to investigate the subjective well-being of ethnic residents in the western regions of China. Before applying causal model analysis, the relationship between variables is analyzed first.

1. Economic development, resident income and the subjective well-being of ethnic residents. To examine the relationships between the level of regional economic development and the subjective well-being of the ethnic residents discussed in this paper, the article divides the western Chinese provinces and autonomous regions into high and low levels of economic development based on their levels of per capita Gross Domestic Product (GDP) as of 2010. In addition, to further study the relationships between resident income gand the subjective well-being of ethnic residents, this article also divides the total household income of residents from the year previous to 2010 into high and low incomes to demonstrate the differences in household income. We have also conducted a contingency analysis of economic development, the income of residents and the subjective well-being of ethnic residents, and presented the analysis results as broken-line graphs (Figs. 3 and 4).

It can be seen from these graphs that the level of economic development has a strong correlation with the subjective well-being of various ethnic groups 


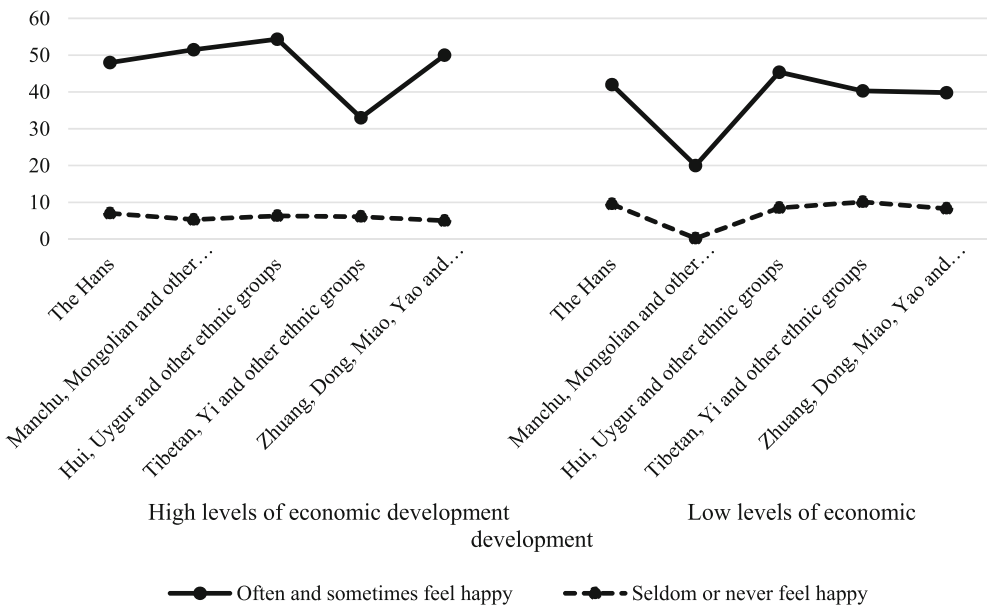

Fig. 3 Levels of regional economic development and subjective well-being of ethnic residents (percentages)

in China, and the level of regional economic development has a stronger impact on the Manchu-Mongol ethnic cluster than on the Hans. In areas with higher levels of economic development, 51.49\% of the Manchu-Mongol ethnic cluster reported that they often feel happy, while in areas with lower levels of economic development, only $20 \%$ of the Manchu-Mongol ethnic cluster reported often feeling happy. In comparison, the subjective well-being of the Hui-Uyghur ethnic cluster in areas with different levels of economic development is evenly distributed. For instance, in areas with higher levels of economic development, $54.35 \%$ of the Hui-Uyghur ethnic cluster often feel happy, while in areas with lower levels of economic development, $45.38 \%$ of this ethnic group reported often feeling happy. In comparison, with regard to the percentage of people from the Hui and Uyghur ethnic cluster who often feel unhappy, the difference between the two regional categories (those with high and low levels of economic development) is only about $1 \%$. Therefore, economic development bears no clear impact on people's subjective well-being in the regions inhabited by the Hui-Uyghur ethnic cluster.

The effect of household income on the subjective well-being is also significant in the Manchu-Mongol ethnic cluster. Around 57.6\% of the ManchuMongol ethnic cluster with higher levels of income reported that they often feel happy, compared to only $40.24 \%$ of low-income residents from this group. Among the Hans and ethnic minority residents such as Tibetans and Yi people, Zhuang and Dong peoples / Miao and Yao peoples, there is a consistent correlation between household income and subjective well-being. As the level of household income increases, the percentage of these ethnic residents who often feel happy also increases, whereas the percentage of people who feel unhappy decreases. Take the families of the Han ethnic group as an example. Around $49.48 \%$ of residents from high-income families report often feeling happy, 


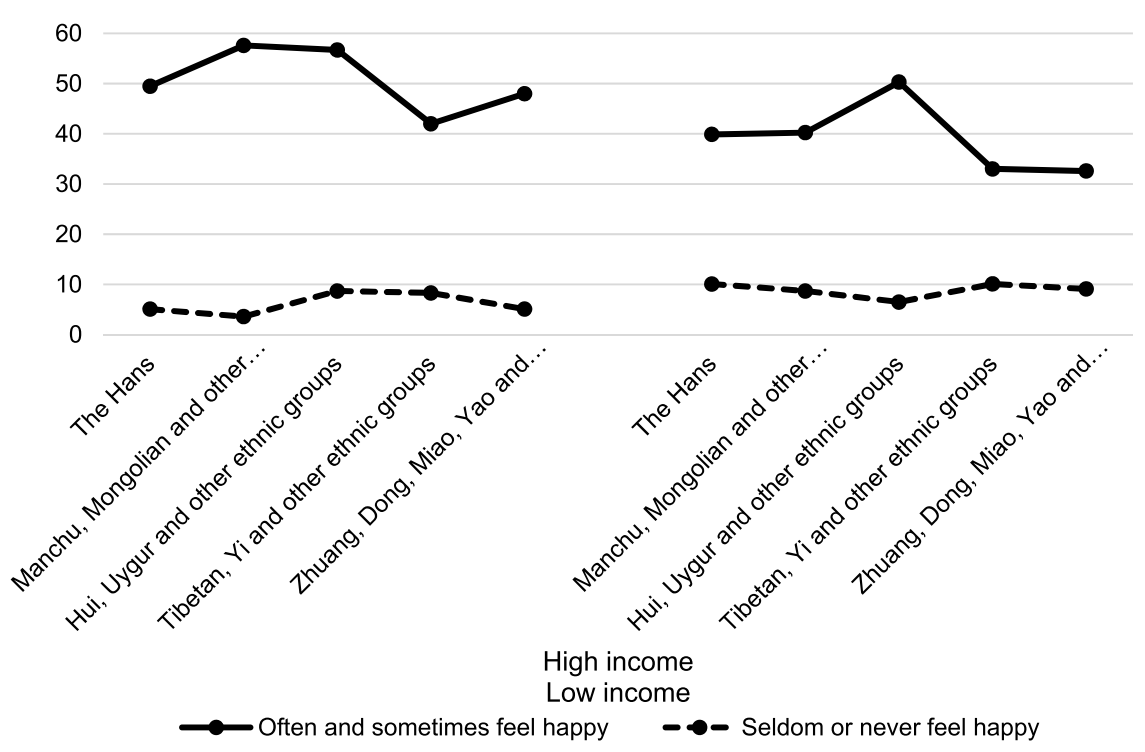

Fig. 4 Household income and subjective well-being of ethnic residents (percentages)

while approximately $5.08 \%$ of residents from high-income families often feel unhappy; meanwhile, about $39.88 \%$ of residents from low-income families often feel happy and $10.09 \%$ of residents from low-income families often feel unhappy. Similar to the effects of regional economic development, the impact of different levels of household income on the subjective well-being of the Hui-Uyghur ethnic cluster is not as clearly defined as that of other ethnic groups.

From this we can see that, on the one hand, a sound economic environment and income are the basic building blocks for the subjective well-being of ethnic residents. As indicators of economic conditions, the level of household income and regional economic development play an irreplaceable role in these residents' subjective well-being. On the other hand, while the variations in the subjective well-being of ethnic residents are largely linked with the variations in economic conditions, it is undeniable that the effects of economic variables on ethnic happiness also do vary.

2. Social integration and the subjective well-being of ethnic residents. In order to analyze the correlation between social integration and the subjective well-being of ethnic residents, this article analyzes the social integration of the Chinese ethnic residents from the perspectives of emotional integration, trustbased integration and integration through communication. In terms of specific measurements, this article measures emotional integration by an individual's marital status, we measure trust-based integration by the status of trust, ${ }^{3}$ and

\footnotetext{
${ }^{3}$ we measure trust-based integration by the amount of trust an individual places in others.
} 


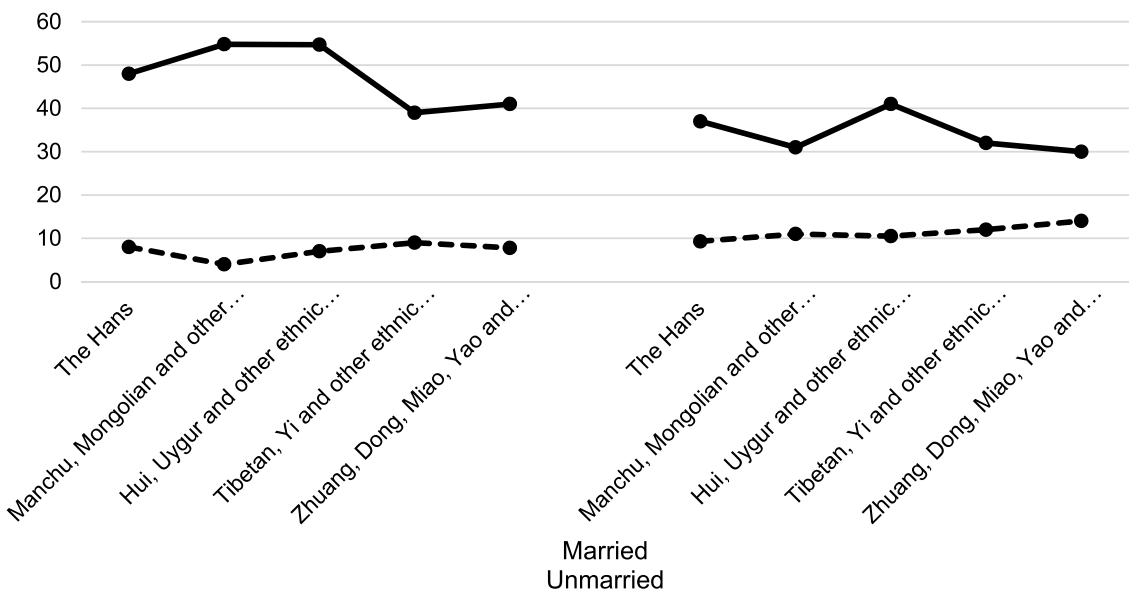

Often and sometimes feel happy

- - Seldom or never feel happy

Fig. 5 Emotional integration and subjective well-being of ethnic groups (percentages)

integration through communication is measured by the percentage of relatives included in an individual's New Year Greeting Network. A lower percentage of relatives in the New Year Greeting Network indicates a higher heterogeneity of social communication, and thus a higher degree of integration; a higher percentage of relatives in the New Year Greeting Network shows a lower heterogeneity of social communication and is thus measured as a lower level of integration. The results of our contingency analysis on emotional integration, trust-based integration, integration through communication and the subjective well-being of ethnic groups are displayed in Fig. 5, Fig. 6 and Fig. 7 as broken-line graphs.

In Fig. 5, the results of our contingency analysis of marital status and the subjective well-being of ethnic residents in the western regions shows that emotional integration has the greatest impact on the subjective well-being of two of our ethnic categories: the Manchu-Mongol ethnic cluster and the HuiUyghur ethnic cluster. Among the Manchu-Mongol ethnic cluster, 54.65\% of married residents often feel happy while $31.43 \%$ of unmarried residents often feel happy. Among the Hui-Uyghur ethnic cluster, $54.83 \%$ of married residents often feel happy, which is $15 \%$ higher than that of unmarried residents; meanwhile, married residents often feel unhappy at about half the rate of unmarried residents. A correlation between marital status and subjective well-being is also found among the Hans and ethnic residents including Tibetans, Yi people, Zhuang and Tong peoples / Miao and Yao peoples, but to varying degrees among people of different ethnic groups.

In Fig. 6, the result of our contingency analysis on integration through trust and the subjective well-being of ethnic groups shows that social trust has a minor effect on the subjective well-being of Han residents, but the difference 

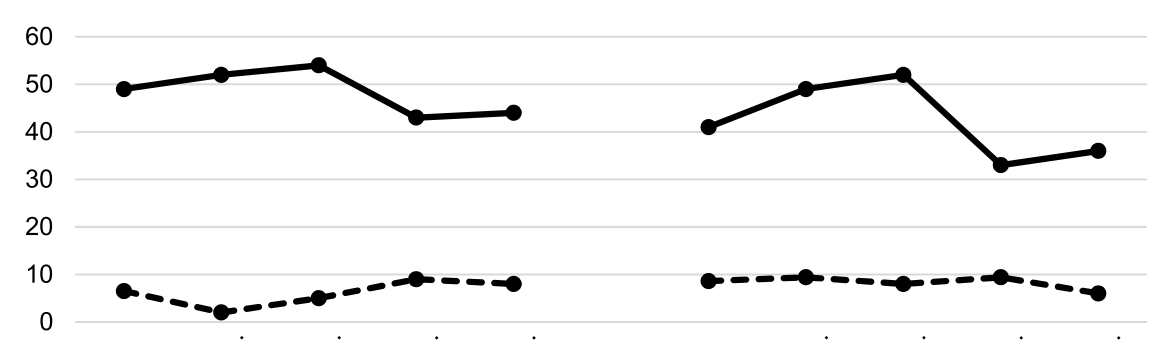

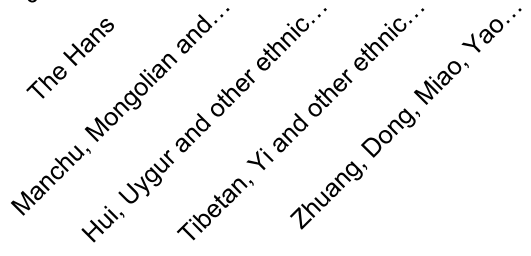

High level of social trust

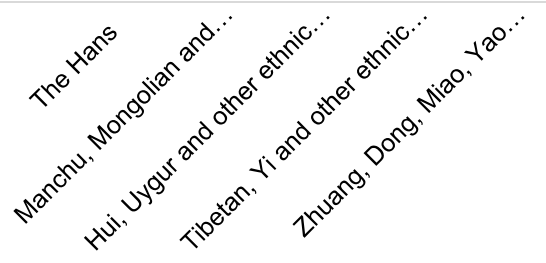

Low level of social

trust

Often and sometimes feel happy $\quad$ - $\quad$ Seldom or never feel happy

Fig. 6 Emotional integration and subjective well-being of ethnic groups (percentages)

is not a significant one. When there is a high level of social trust, $49.58 \%$ of Han residents often feel happy while $6.20 \%$ often expressed unhappiness; when the level of social trust is low, $41.19 \%$ of Han residents often feel happy but $8.26 \%$ often feel unhappy. The impact of distrust on the negative emotional experience of the Manchu-Mongol ethnic cluster is more pronounced than that

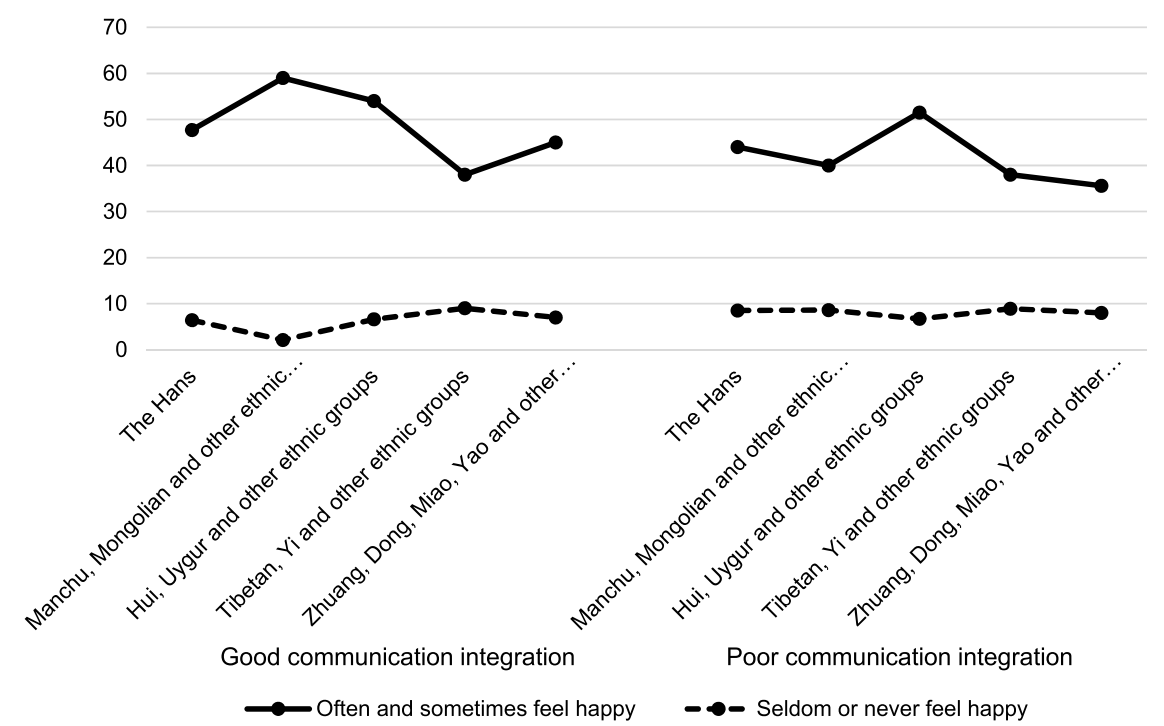

Fig. 7 Integration through communication and subjective well-being of ethnic groups (percentages) 
on the Han ethnic group. However, variations in the levels of social trust have little effect on the subjective well-being of ethnic residents such as Huis and Uyghurs. Meanwhile, a certain degree of correlation between the subjective well-being and integration through trust is found among the Manchu-Mongol ethnic cluster, Tibetans and Yi people, Zhuang and Dong peoples / Miao and Yao peoples. However, on the whole, the impact of the trust-based integration on the subjective well-being of different ethnic groups is not as pronounced as the impact of marriage, which forms part of emotional integration.

Integration through communication, which is measured based on the degree of diversity in an individual's communication, is another key factor for testing the degree of social integration. As is shown in Fig. 7, the results of the correlation analysis between integration through communication and the subjective well-being of ethnic groups shows that integration through communication has the most pronounced influence on the subjective well-being of the ManchuMongol ethnic cluster. As many as $58.82 \%$ of Manchus and Mongolians often feel happy when they measured higher for integration through communication, while only $39.77 \%$ of this group often feel happy when integration through communication is not achieved as desired. In addition, the data shows that changes in integration through communication have little impact on the subjective well-being of the following two ethnic categories: Huis and Uyghurs, and Tibetans and Yi people. This minor impact shows that social communication and integration has different effects on the subjective well-being of different ethnic groups.

It can be said that, in all three dimensions, social integration are correlated with the subjective well-being of different ethnic residents, but to varying degrees. People with different ethnic cultures experience these interactions in different ways. For people of some ethnic groups with unique religious beliefs, cultures or histories, a high degree of homogeneity of communication probably represents a high recognition of ethnic culture. The impact of integration through communication on the subjective well-being of ethnic groups should be studied against the backdrop of multi-ethnic cultures.

\section{Analysis and results}

The result of the contingency analysis shows that the residents of different ethnic groups in the western regions of China have the subjective well-being on the whole, but to varying degrees. As the core explanatory variables in this article, both economic development and social integration impact the subjective well-being in the western regions of China to varying degrees. However, what impact do these two variables exert independently of each other, and what are the differences between their impacts on each different ethnic group? Below, the article will provide further analysis on the causal relationships between economic development, social integration, and the subjective well-being of ethnic groups by means of an empirical model. 


\section{Choice of variables}

In order to make our theoretical analysis, we have selected the following main research variables: subjective well-being, ethnic group, household income, regional economic development, and social integration.

1. Dependent variable: subjective well-being. The degree of subjective wellbeing of ethnic residents in the western region is measured in light of the answers given to the question "Did you feel happy last week?" in the questionnaire, and values are assigned to different answers on a scale of 1-5.

2. Independent variables. First, ethnic group. In the process of exploring the subjective well-being of ethnic groups, a direct overall comparison between the subjective well-being of the Hans and the subjective well-being of all other ethnic minority groups may lead us to ignore the differences between individual ethnic groups in terms of culture and regional development. As a result, we would see shortcomings such as simplified inferences and one-sided research results may appear. The criterion and method used above to classify ethnic groups was also applied here. A total of 28 ethnic groups included in the survey samples were categorized into four groups of people: Manchus and Mongolians, Huis and Uyghurs, Tibetans and Yi people, and Zhuang and Dong peoples / Miao and Yao peoples.

Second, economic development. Economic development is used to examine the level of household income and the level of regional economic development. The household income is the most direct embodiment of a family's economic status. In this article, household income is used as an indicator of microeconomic development, with 10,000 yuan per month as the unit of measurement. Meanwhile, based on the provincial data from the National Bureau of Statistics (see website), the 2010 per capita GDP of the western provinces were divided into high, middle, and low levels to reflect the levels of economic development of the western regions inhabited by ethnic residents.

Third, social integration. In existing studies, social integration has been measured from subjective and objective perspectives (Yue, 2009). An individual's marital status and their level of trust in others give and indication as to their subjective emotions and cognition, and so belong to the subjective dimension of our social integration variable. The heterogeneity of communication effectively represents the specific status of an individual's social interactions and social networks, and so belongs to the objective dimension of our social integration variable. This article uses three variables of emotional integration, trust-based integration, and integration through communication to measure the degree of social integration of residents in the western regions of China. Emotional integration is measured by assigning a value of 1 to married residents and 0 to unmarried residents and all others. Trust-based integration is measured by assigning a value of 1 to residents with a high degree of trust, represented by answers of "full trust" and "relatively high trust" in the questionnaire, and a value of 0 to those with a low degree of trust, represented by the answers "unclear," "relatively low trust," and "no trust at all." In terms of integration through communication, the percentage of relatives in an individual's New Year Greeting Network is used to reflect the degree of heterogeneity of social communication. In other words, the higher the percentage of relatives in the New Year Greeting Network, the lower the degree of heterogeneity in social communication. 


\section{Control variables mainly include gender, age, years of education, self-assessment, and health status}

\section{Descriptive statistics for variables (Table 3)}

Table 3 Variable descriptive statistics $(N=10,098)$

\begin{tabular}{|c|c|c|c|c|c|}
\hline Variable & $\begin{array}{l}\text { Mean/ } \\
\text { percentage }\end{array}$ & $\begin{array}{l}\text { Standard } \\
\text { deviation }\end{array}$ & $\begin{array}{l}\text { Minimum } \\
\text { value }\end{array}$ & $\begin{array}{l}\text { Maximum } \\
\text { value }\end{array}$ & Note \\
\hline \multicolumn{6}{|l|}{ Subjective well-being } \\
\hline Often & $44.74 \%$ & & & & Dependent variable \\
\hline Sometimes & $35.48 \%$ & & & & \\
\hline Seldom & $12.39 \%$ & & & & \\
\hline Never & $7.39 \%$ & & & & \\
\hline Gender & $50.98 \%$ & & 0 & 1 & $0=$ female $; 1=$ Male \\
\hline Age & 44.83 & 14.63 & 18 & 93 & \\
\hline Education (Years) & 8.03 & 4.50 & 0 & 19 & \\
\hline Self-evaluation & $81.98 \%$ & & 0 & 1 & $0=$ negative $; 1=$ positive \\
\hline Health condition & $64.17 \%$ & & 0 & 1 & $0=$ worse $; 1=$ better \\
\hline \multicolumn{6}{|l|}{ Ethnicity } \\
\hline Han & $72.32 \%$ & & & & $1=$ Hans \\
\hline $\begin{array}{l}\text { Manchu, Mongolian } \\
\text { and other ethnic } \\
\text { groups }\end{array}$ & $2.05 \%$ & & & & $\begin{array}{l}2=\text { Manchu, Mongolian and } \\
\text { other ethnic groups }\end{array}$ \\
\hline $\begin{array}{l}\text { Hui, Uygur and other } \\
\text { ethnic groups }\end{array}$ & $7.65 \%$ & & & & $\begin{array}{l}3=\text { Hui, Uygur and other } \\
\text { ethnic groups }\end{array}$ \\
\hline $\begin{array}{l}\text { Tibetan, Yi and other } \\
\text { ethnic groups }\end{array}$ & $12.42 \%$ & & & & $\begin{array}{l}4=\text { Tibetan, Yi and other } \\
\text { ethnic groups }\end{array}$ \\
\hline $\begin{array}{l}\text { Zhuang, Dong, Miao } \\
\text { and Yao and other } \\
\text { ethnic groups }\end{array}$ & $5.65 \%$ & & & & $\begin{array}{l}5=\text { Zhuang, Dai, Miao and } \\
\text { Yao and other ethnic } \\
\text { groups }\end{array}$ \\
\hline Family income & 2.94 & 6.06 & 0 & 300 & \\
\hline \multicolumn{6}{|c|}{ Regional economic development level } \\
\hline High & $36.14 \%$ & & & & \\
\hline Medium & $33.50 \%$ & & & & \\
\hline Low & $30.36 \%$ & & & & \\
\hline Emotional integration & $82.85 \%$ & & 0 & 1 & $\begin{array}{l}0=\text { unmarried/other; } 1= \\
\text { married }\end{array}$ \\
\hline Trust integration & $48.09 \%$ & & 0 & 1 & $\begin{array}{l}0=\text { high degree of trust; } 1= \\
\text { low degree of social trust }\end{array}$ \\
\hline $\begin{array}{l}\text { Communication } \\
\text { integration }\end{array}$ & $63 \%$ & 0.23 & 0 & 1 & $\begin{array}{l}\text { The percentage of relatives } \\
\text { in the New Year Greeting } \\
\text { Network }\end{array}$ \\
\hline
\end{tabular}




\section{Analytical results of data and research findings (Table 4)}

Table 4 Model analysis of economic development, social integration and the happiness of ethnic groups

\begin{tabular}{|c|c|c|c|}
\hline \multirow[t]{2}{*}{ Variable } & (1) & (2) & (3) \\
\hline & $\begin{array}{l}\text { Baseline } \\
\text { model }\end{array}$ & $\begin{array}{l}\text { Economic development } \\
\text { model }\end{array}$ & $\begin{array}{l}\text { Social integration } \\
\text { model }\end{array}$ \\
\hline Gender & $-0.089 * *$ & $-0.089 * *$ & $-0.103 * * *$ \\
\hline Age & $0.012 * * *$ & $0.012 * * *$ & $0.009 * * *$ \\
\hline Degree of education & $0.030 * * *$ & $0.025 * * *$ & $0.022 * * *$ \\
\hline Self-evaluation & $1.164 * * *$ & $1.153 * * *$ & $1.128 * * *$ \\
\hline Health condition & $0.578 * * *$ & $0.547 * * *$ & $0.531 * * *$ \\
\hline \multicolumn{4}{|l|}{ Ethnicity (refer to the Hans) } \\
\hline $\begin{array}{l}\text { Manchu, Mongolian and other ethnic } \\
\text { groups }\end{array}$ & 0.154 & 0.071 & 0.046 \\
\hline Hui, Uygur and other ethnic groups & $0.323 * * *$ & $0.340 * * *$ & $0.317 * * *$ \\
\hline Tibetan, Yi and other ethnic groups & $-0.138 * *$ & -0.011 & 0.002 \\
\hline $\begin{array}{l}\text { Zhuang, Dong, Miao and Yao and other } \\
\text { ethnic groups }\end{array}$ & -0.213 & -0.132 & -0.145 \\
\hline Family income & & $0.018 * * *$ & $0.016 * * *$ \\
\hline \multicolumn{4}{|l|}{ Regional economic development level } \\
\hline Medium & & $0.186^{* * *}$ & $0.179 * * *$ \\
\hline High & & $0.361 * * *$ & $0.348 * * *$ \\
\hline Emotional integration & & & $0.394 * * *$ \\
\hline Trust integration & & & $0.231 * * *$ \\
\hline Communication integration & & & $-0.317 * * *$ \\
\hline Bayesian information criterion & $22,940.82$ & $22,900.76$ & $22,819.46$ \\
\hline Sample size & 10,098 & 10,098 & 10,098 \\
\hline
\end{tabular}

Note: $* * * \mathrm{p}<0.01,{ }^{*} \mathrm{p}<0.05,{ }^{*} \mathrm{p}<0.1$ Baseline model 1 explains the effect of control variables

In general, a significant gender difference exists in the subjective well-being of western residents. The negative coefficient for gender indicates that male residents have a lower subjective well-being than females. Further, as age and the level of education increase, residents are likely to feel happy more often. Meanwhile, a positive self-assessment and a sound health status can have a relatively significant impact upon subjective well-being. Subjective well-being varies between different ethnic groups. The Hui-Uyghur ethnic cluster feel happy more often than the Hans, while ethnic residents including Tibetans and Yi people or ethnic residents including Zhuang and Dong peoples / Miao and Yao peoples feel happy less often than the Hans. There is no significant difference between the Hans and the Manchu-Mongol ethnic cluster in terms of subjective well-being. 
In order to test the impact of economic factors on the subjective well-being of ethnic residents, we have established Model 2 with two more variables (the level of household income and the level of regional economic development) added to those of Model 1. The analysis result shows that both the level of household income and the level of regional economic development are significantly correlated with the subjective well-being of residents in the western regions. For individuals, as household income increases, their subjective wellbeing also increases. Across different regions, residents living in an area with the middle level of economic development are likely to feel happy more often than those living in regions with the lower level of economic development, while residents of a region with a high level of economic development are likely to feel happy the most often. Compared with Model 1, the variations in subjective well-being between people of different ethnic groups have changed. For instance, the variations in the subjective well-being between the Hans and people of other ethnic minorities such as Tibetans and Yi people, Zhuang and Dong peoples / Miao and Yao peoples have disappeared using Model 1, indicating that household income and regional economic development can effectively bridge the gap in subjective well-being between the Hans and the people of these ethnic minorities. In other words, the variations in economic variables largely account for the variations in subjective well-being between the Hans and people of the abovementioned ethnic minorities. In Model 2, however, the Huis and Uyghurs are still likely to feel happy more often than the Hans, indicating that economic variables have little effect on the variations in subjective well-being between the Hans and ethnic residents such as Huis and Uyghurs. No significant difference in subjective well-being is found between the Hans and the Manchu-Mongol ethnic cluster. It can be seen that reducing the gap in economic development between the eastern and western regions, particularly strengthening economic development in the southwestern region inhabited by ethnic residents including Tibetans and Yi people, and Zhuang and Dong peoples / Miao and Yao peoples, has major, direct significance on enhancing the subjective well-being of the people of these ethnic groups, and further enhancing solidarity and integration among these ethnic residents. The research by Yang Jingchu also suggests that narrowing the gap between eastern and western development in China is of major significance on strengthening the solidarity and integration between the Hans and people of ethnic minorities (Yang, 1997). The impact of economic factors described above goes some lengths to verify the hypothesis of economic development in most ethnic minority areas in the western regions of China.

Model 3 is used to test the impact of social integration on the subjective wellbeing of ethnic residents. Three variables-marital status, social trust, and heterogeneity of communication-are added to variables of Model 2 to evaluate the specific impacts of emotional integration, trust-based integration, and integration through communication on the subjective well-being of ethnic residents. The results of our data analysis show that the impacts of these three variables of social integration are all significant, which proves that social integration can effectively improve the subjective well-being of residents in ethnic regions. Of all these 
variables, emotional integration, measured by marital status, exerts an influence on all ethnic groups, as the probability that married residents often feel happy is generally greater than that of unmarried residents. Likewise, social trust shows a significant positively correlation with the subjective well-being of residents, as the higher the social trust, the more likely the residents were to feel happy often. In terms of social communication, as the percentage of relatives in a given social network increases, the subjective well-being of residents declines. This negative correlation shows that the more the residents communicate with people outside of their own families and relatives, the greater subjective well-being they will have. Such a negative correlation also empirically shows that social support networks, social acceptance, and social identity have an impact upon the subjective wellbeing of all ethnic residents.

It is also of note that, in terms of the variations found in the subjective wellbeing of ethnic residents, all three models show a special phenomenon that HuiUyghur ethnic cluster probably feel happy more often than the Hans and people of other ethnic minorities. In addition, economic variables and social integration variables do not have a significant impact on the Hui-Uyghur ethnic cluster. Such a phenomenon indicates that some other endogenous variables specific to people of these ethnic groups influence their subjective well-being. Further discussion in this respect is needed in future studies.

\section{Discussion and conclusions}

First, the people of all ethnic groups in the western regions of China generally feel happy, as the residents who indicated that they often feel happy far exceed those who did not. In addition to demographic factors such as gender, education level, and health, the subjective well-being of ethnic residents in the western regions of China is affected by such factors as the level of household income and regional economic development, as well as by social integration factors such as marital status, social trust, and heterogeneity of communication. Different ethnic groups vary in their subjective well-being. Although economic variables significantly impact on the subjective well-being of people of most ethnic groups, they have little impact on the subjective well-being of ethnic groups including Huis and Uyghurs.

Second, previous studies have had a tendency towards the pre-established hypothesis that people of the Chinese ethnic minorities have a lower subjective wellbeing than the Hans since people of ethnic minorities are affected by factors like geographical environment, population size, and cultural influences. However, such a hypothesis is not tenable seen from the results of the survey data collected from 12 western Chinese provinces, direct-administered municipalities and autonomous regions. The data proves that there is no significant variation in subjective wellbeing between the Hans and the Manchu-Mongol ethnic cluster, and that the HuiUyghur ethnic cluster feel happy more often than the Hans. Such a variation has been statistically significant in all three models. Moreover, residents of various ethnic groups in China do not vary greatly in their subjective well-being. Therefore, 
the assertion that the Hans enjoy greater subjective well-being than people of ethnic minorities is unfounded.

Third, the effect of the Easterlin Paradox has been proven to varying degrees. For the Tibetan-Yi ethnic cluster and ethnic residents including Zhuang and Dong peoples / Miao and Yao peoples, improving the economic situation is very important for satisfying the needs of these residents, since they are still relatively underdeveloped and financially disadvantaged. According to the Easterlin Paradox, increased income has a greater effect on the subjective well-being of people in poor regions and countries than people in more economically developed regions and countries. Therefore, economic development and income growth should significantly improve the subjective well-being of ethnic groups in these underdeveloped areas. In fact, the results of our data analysis using the three models show that the subjective well-being of ethnic residents including Tibetans and Yi people, Zhuang and Dong peoples / Miao and Yao peoples is consistent with the logic of the Easterlin Paradox.

Finally, the impacts of control variables such as health, level of education and age on the subjective well-being are consistent with the research findings of other scholars. In other words, all ethnic residents in the western regions of China will have greater subjective well-being with better health, higher levels of education, and increased age.

The subjective well-being of ethnic residents expresses how residents from every Chinese ethnic group feel about and evaluate their own lives. People's subjective well-being bears a substantial influence on the harmonious development of ethnic minority groups in China, as well as on the solidarity among these groups. Based on the above research conclusions, the article puts forward the following two policy suggestions to further improve and increase the subjective well-being of ethnic groups.

First, economic factors remain pivotal to the overall improvement of subjective well-being for the ethnic groups living in the less developed ethnic regions in the west of China. Moreover, health and the level of education also remain important factors that can restrict the subjective well-being of an individual. Underdeveloped economies and insufficient provision of education and medical resources hinder the improvement of subjective well-being for ethnic residents in the western regions. Therefore, the major means by which we can improve ethnic residents' subjective well-being on the whole are to accelerate economic development and urbanization in the western regions, and promote the construction of more medical and educational resources there. In particular, increased support must be provided to the Tibetan-Yi ethnic cluster, Zhuang and Dong peoples / Miao and Yao peoples, who live in economically underdeveloped regions where subjective well-being is relatively lower.

Second, social integration opens a new path for improving the subjective wellbeing of ethnic groups. While emphasizing the importance of national culture and values, efforts should be made to respect the distinctive cultures and traditions of ethnic minorities and enhance the subjective well-being of ethnic minority residents by enabling them to have a sense of cultural belonging. Meanwhile, social trust and external communication are significantly correlated with the subjective well- 
being of people from every ethnic group in the western regions of China. Governments at the grass-root level can take initiative in advocating and organizing various forms of social and cultural activities to promote interactions and integration between different ethnic groups, so as to form a more open environment where people of different ethnic groups are willing to take action and communicate with each other. In addition, efforts should also be made to promote the concept of "honesty and love, solidarity and mutual assistance" on relationships between ethnic groups, and increase the level of social trust and subjective well-being of residents by creating an equitable and harmonious interpersonal environment.

Authors'contributions Jianke Yang: Determine the topic selection and framework of the thesis, and complete the main work of the thesis writing. Qi Wang: Data analysis, drawing and table making of the paper. The author(s) read and approved the final manuscript.

Availability of data and materials Available from the author upon request.

\section{Declarations}

Ethics approval and consent to participate Not applicable.

Consent for publication Not applicable.

Competing interests We have no any competing interests.

Open Access This article is licensed under a Creative Commons Attribution 4.0 International License, which permits use, sharing, adaptation, distribution and reproduction in any medium or format, as long as you give appropriate credit to the original author(s) and the source, provide a link to the Creative Commons licence, and indicate if changes were made. The images or other third party material in this article are included in the article's Creative Commons licence, unless indicated otherwise in a credit line to the material. If material is not included in the article's Creative Commons licence and your intended use is not permitted by statutory regulation or exceeds the permitted use, you will need to obtain permission directly from the copyright holder. To view a copy of this licence, visit http:// creativecommons.org/licenses/by/4.0/.

\section{References}

Bian, Y., L. Zhang, Yang, et al. 2014. Subjective wellbeing of Chinese people: A multifaceted view. Social Indicators Research 121: 75-92. https://doi.org/10.1007/s11205-014-0626-6.

CCTV. 2017. New findings from large-scale survey:Those earning an annual income of more than 300,000 Yuan may not necessarily feel Happy.http://jingji.cctv.com/2017/03/ 07 / ARTIm9mcDt45GrnUlAafEwob170307.Shtml. .

Diener, E., and R. Biswas Diener. 2002. Will money increase subjective well-being. Social Indicators Research 57: 119-169. https://doi.org/10.1023/A:1014411319119.

Diener, E., S. Oishi, and R.E. Lucas. 2003. Personality, culture, and subjective well-being: Emotional and cognitive evaluations of life. Annual Review of Psychology 54: 403-425. https://doi.org/10.1146/a nnurev.psych.54.101601.145056.

Easterlin, Richard A., et al. 2010. The happiness-income paradox revisited. Proc Natl Acad Sci USA 107: 22463-22468. https://doi.org/10.1073/pnas.1015962107.

Guo, Xueping, Feng Chao, and Zhang Honglei. 2008. Research on the relationship between subjective well-being and personality of middle school teachers of different ethnic groups. J Shijiazhuang Univ 10: 99-103.

Huang, Kuang and Ga Rida. 2010. Review of social integration theory research. Expanding horizons 6:8688.Doi: CNKI:SUN:XISY.0.2010-06-030. 
Ji, Yongbao, Jingyun Gao, and Yang Jun. 2016. The impact of the social integration of migrants on their subjective well-being: Taking Shandong Province as an example. Urban Problems 7: 95-103.

Klein, Carlo. 2013. Social capital or social cohesion: What matters for subjective well-being? Social Indicators Research 110: 891-911. https://doi.org/10.1007/s11205-011-9963-x.

Liu, Junqiang, Xiong Moulin, and Su Lin. 2012. Subjective well-being of nationals in the period of economic growth. Soc Sci China: 82-102.

Neff, Daniel F. 2007. Subjective well-being, poverty and ethnicity in South Africa: Insights from an exploratory analysis. Soc Indicators Res 80: 313-341.

Saunders, L.L., R. Gregory-Bass, and J.S. Krause. 2013. Gender, race, pain, and subjective well-being of adults with spinal cord injury. J Health Care Poor Underserved 24: 1257-1265. https://doi.org/10.1353/ hpu.2013.0119.

Spiers, Andrew, Walker, Gordon, \& J. 2009. The effects of ethnicity and leisure satisfaction on happiness, peacefulness, and quality of life. Leisure Sciences. 31:84-99. doi:https://doi.org/10.1080/01490400802 558277. China statistics bureau database. 2010. http://data.stats.gov.cn/search.htm?s=201 0percentE5percentB9percentB4percentE5percent90percent84percentE7percent9Cpercent81GDP. Accessed 8 July 2017.

Xing, Zhanjun. 2011. Research on the relationship between Residents' income and subjective well-being in China. Sociology study 1:196-219.Doi: CNKI:SUN:SHXJ.0.2011-01-009.

Xiong, Moulin, and Jiang Lihua. 2014. Ethnic integration from the perspective of subjective well-being of ethnic groups: A study based on CGSS data. J Southwest Univ Nationalities 7: 59-64.

Yang, Chaojie, et al. 2015. Analysis of the Subjective well-being of Residents in Ethnic Minority Compact Communities and Its Influential Factors -Based on a Survey in Lanzhou City. Journal of Arid Land Resources and Environment 12:31-36.doi:https://doi.org/10.13448/j.cnki.jalre.2015.396.

Yang, Jingchu. 1997. On narrowing the development gap between the eastern and Western regions and strengthening the solidarity and cooperation between the Han and ethnic minorities. Ethno-Natl Stud 3: 23-33.

Yue, Zhongshan, Du Haifeng, Li Shuzheng, and Feldman. 2009. Concepts, Theories, and Applications of Contemporary Western Social Integration Studies. Journal of Public Management 6:114-128.doi: CNKI: SUN:GGGL.0.2009-02-018.

Zhang, Pengcheng. 2008. Research on subjective well-being of ethnic minority college students. J Guizhou Minzu Univ 108: 177-180. https://doi.org/10.3969/j.issn.1003-6644.2008.02.044.

Zhao, Changlin. 2011. Empirical analysis of factors influencing the subjective well-being of residents in ethnic minority areas of Yunnan Province. J Yichun Univ 6: 36-38. https://doi.org/10.3969/j.issn.1671-3 80X.2011.06.013.

Zhou, Mo, Jingli Mao, et al. 2007. The subjective well-being of ethnic minority college students and its influential factors. Chinese J Health Psychol 15: 312-314. https://doi.org/10.3969/j.issn.1005-1252.2007.04.009.

\section{Comments}

Publisher's Note Springer Nature remains neutral with regard to jurisdictional claims in published maps and institutional affiliations. 